\title{
Mesenchymal stem cells in systemic sclerosis therapy
}

\author{
Daniel Witold Dudek ${ }^{1}$, Ewa Walczuk², Anna Wajda², Agnieszka Paradowska-Gorycka ${ }^{2}$ \\ ${ }^{1}$ Biostructure Center of the Medical University of Warsaw, Poland \\ ${ }^{2}$ Department of Molecular Biology, National Institute of Geriatrics, Rheumatology and Rehabilitation, Warsaw, Poland
}

\begin{abstract}
Systemic sclerosis (SSc) is a rare autoimmune disorder with a high mortality rate. There are still many unknowns concerning the pathophysiology of this disease, due to its clinical heterogeneity. Since there is still no curative treatment, researchers focus on finding novel methods to help the patients. One of the valid options is cellular therapy, and mesenchymal stem cells (MSCs)-based therapy yields great expectations. These cells possess especially valuable attributes regarding key points of SSc. Nevertheless, the effectiveness and safety of this therapy must undergo a rigorous process of verification.

In preclinical trials, animal models proved to be a valuable source of scientific knowledge regarding SSc. Because of that, it has been possible to test autologous or allogeneic MSCs from various sources in many clinical trials. A lot of aspects still have to be determined to assess their potential in the management of SSc, probably in association with other therapies.
\end{abstract}

Key words: systemic sclerosis, mesenchymal stem cells, cell therapy.

\section{Introduction}

Systemic sclerosis (SSc) is a rare, chronic and autoimmune connective tissue disorder, mostly affecting middle-aged patients $[1,2]$. The prevalence of this disease ranges from 100 to 300 per million, depending on the country [3].

The typical classification consists of two types of SSC - limited or diffused, based on the extent and distribution of skin fibrosis [4, 5]. Although the background of this disease has been studied for many years, the pathophysiology of SSc is still not completely understood.

As of now, the characteristics of this disease are based on the three main pathologies, including vascular damage, fibrosis and immune abnormalities. Systemic sclerosis affects mostly skin and lung tissues; however, the heart and digestive tract can also be involved [1].

All of this leads to psychological and functional disabilities, which furthermore lower the quality of life and reduce life expectancy $[4,6,7]$. Patients with SSc die mostly due to disorders related to the disease itself where almost half of them develop a higher probability of cardiovascular incidents in comparison with the gene- ral population [8]. So far, no curative treatment is available. Instead, a common solution proposed to patients is symptomatic immunotherapy, focused on alleviating pain and improving functional abilities [9].

However, there are numerous novel strategies intended to treat SSc. Among them, there is mesenchymal stem cells (MSCS)-based therapy, whose safety and effectiveness are currently being evaluated. Therefore, this paper aims to review recent research on MSCsbased cell therapies in SSC.

\section{Mesenchymal stem cells and stromal vascular fraction}

Mesenchymal stem cells are adult, multipotent progenitor cells, which have been identified and described in several tissues, such as adipose-derived MSCS (ASCS), bone marrow-derived MSCS (BM-MSCS) and placenta (PL-MSCS) [10]. The most important characteristic of MSCs is their pleiotropic activity, which is thoroughly investigated for application in many pathological conditions [11, 12]. It is the specifically proangiogenic, antiinflammatory and immunomodulatory properties of 
MSCs which make them great candidates for targeted cell-based therapy [13].

Up until now, patients have been enlisted in hundreds of clinical trials (Fig. 1), where the safety of MSCS injections was verified. Meta-analyses show fewer complications after MSCs implantation in comparison to standard care procedures [14]. However, it is worth noting that these analyses also reveal a significant increase in occurrence of post-injection fever [15]. This can be a potential problem for the future usage of MSCs. Regardless, the beneficial effects of MSCs-based therapy were demonstrated in patients with systemic scleroderma as well.

Adipose tissue is considered a type of connective tissue that exhibits morphological, functional, and regulatory heterogeneity. It is not only a satisfactory source of adipose-derived MSCs, but it also contains various other cells, including mature cells (fibroblasts, smooth muscle and blood cells) and progenitor cells (preadipocytes, vascular and hematopoietic progenitors) [16].

All of them can be collectively labeled as the stromal vascular fraction (SVF) [17]. It has been discovered that the SVF possesses anti-inflammatory and regenerative properties in damaged tissues, mostly due to its ability to induce the secretion of growth factors [18]. This attribute attracts significant attention for potential use in various fields, such as orthopedics, internal medicine and wound healing.

The efficacy of MSCs is related to the secretion of different bioactive molecules, enabling them to communicate with the environment in multiple ways [19]. Among these molecules are cytokines, chemokines, growth factors and extracellular vesicles (EVs). Extracellular vesicles are differently shaped, membrane-surrounded structures, which contain mRNA, miRNA, proteins and mitochondrial fragments. These are involved in the regulation of many routes of signaling pathways and represent a relevant component of the regenerative function of MSCs as they participate in immune modulation and tissue repair [20].

Extracellular vesicles are released from different cells and can serve as biomarkers and contribute to eliminating unwanted components from the environment. All of these mentioned factors are responsible for regulating endogenous cell activity. Their identification led to a better understanding of the interactions between MSCs and the other bioactive molecules connected with fibrosis, vasculopathy and autoimmunity (Fig. 2).

In regards to MSCs-based therapies, the aspect of their tumorigenicity should also be taken into consideration. This risk is widely discussed in the literature, and the best way to summarize all the statements is to say that MSCS represent a dualistic nature in regards to tu-

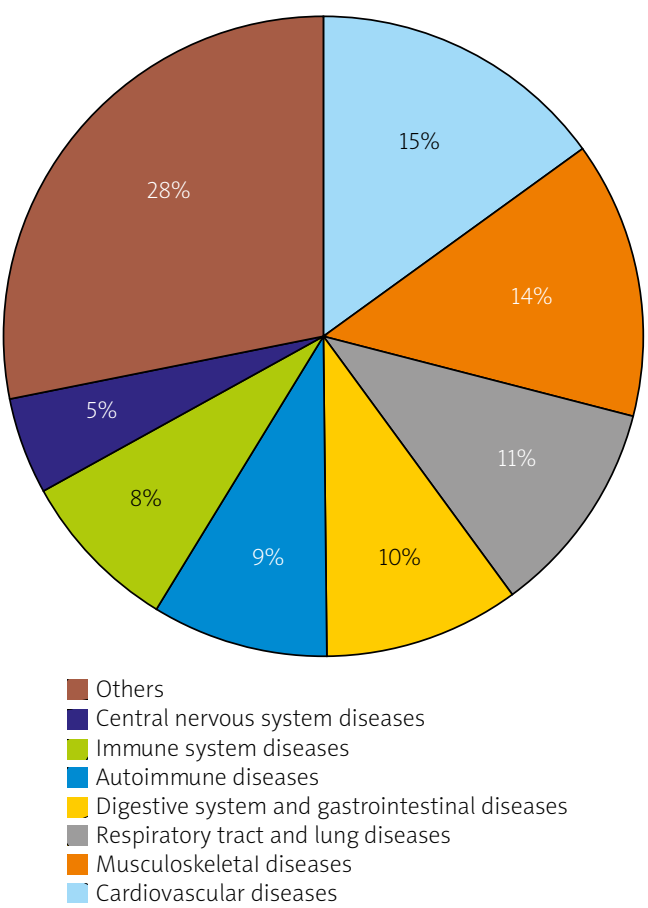

Fig. 1. The chart represents the percentage share of the groups of individual pathological conditions from all of the currently registered mesenchymal stem cell-based therapies (199 studies). Only ongoing and completed studies from early phase 1 to phase 4 were included. Data obtained from ClinicalTrials.gov.

morigenesis. While some studies have shown their antitumorigenic effects, others compare stem cells to tumor cells, because of their ability to proliferate for a long time, high viability, and resistance to apoptosis [21].

Many factors can affect the tumorigenesis potential at different stages of transplantation, including donor's age, growth regulators expressed by recipient tissue, and mechanisms controlling the behavior of the MSCS at the target site $[22,23]$. Also, the potential risks of long-term post-transplantation immunosuppressive therapy associated with the MSCs are a central part of this assessment [24].

However, some factors secreted by MSCs may have antitumor properties. For instance, Clarke et al. [25] reported that breast cancer cells cultured in MSCs-conditioned medium exhibit significant migratory inhibition compared with cells cultured in a standard medium.

Furthermore, the inhibition of tumor cell growth was also shown in a study by Bruno et al. [26], where different cancerous cell lines co-cultured in the presence of BM-MSCs exhibited reduced in vitro growth. To sum up, there is no obvious answer regarding the potential of MSCs in tumorigenesis. 


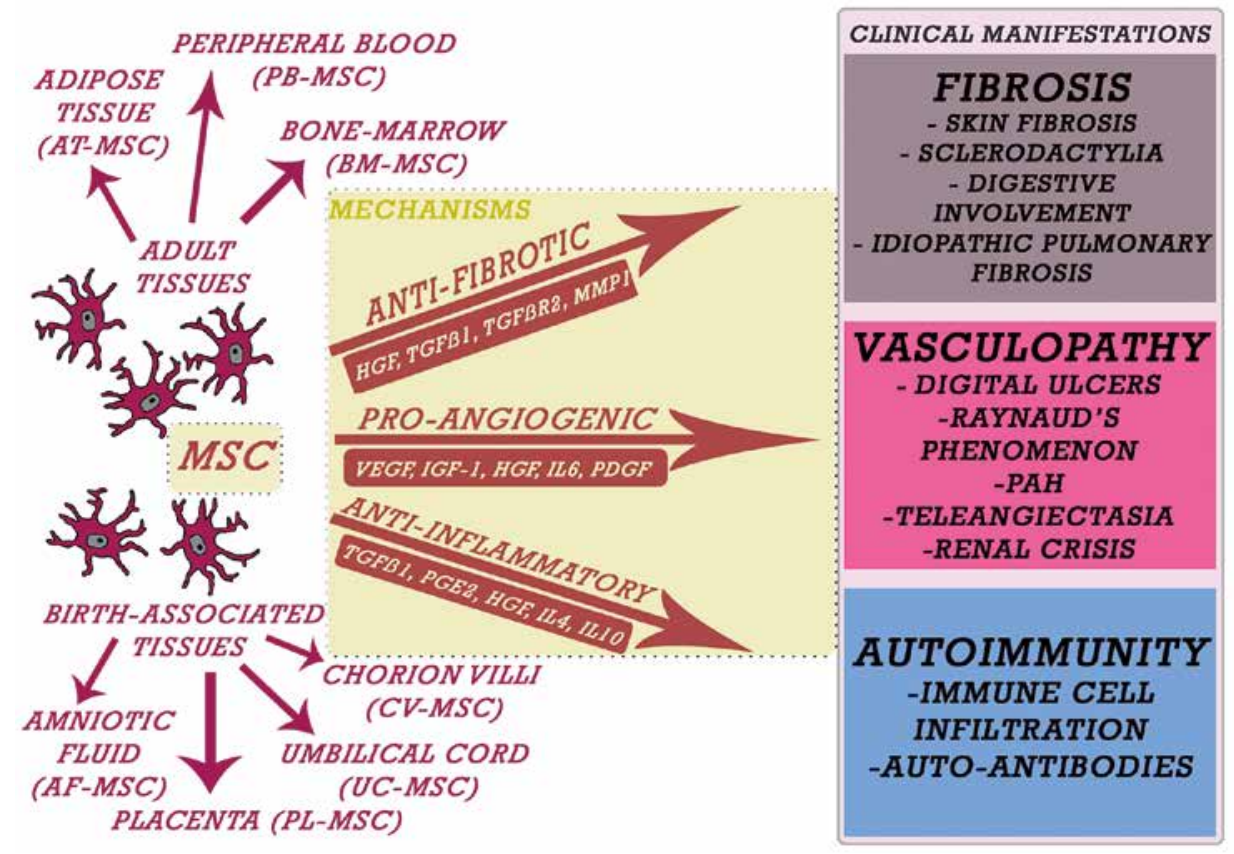

Fig. 2. Different mechanisms of activity of mesenchymal stem cells in systemic sclerosis. Stem cells from adult tissues and birth-associated tissues display various functions, which participate in the treatment of systemic sclerosis via the release of mediators (squares under arrows). These mediators are associated with anti-fibrotic, proangiogenic, and anti-inflammatory functions and correspond with the improvement in specific clinical manifestations (colored boxes).

\section{Role of mesenchymal stem cells in preclinical models of systemic sclerosis and encountered problems}

To evaluate the potential role in SSc treatment, MSCS were used in numerous preclinical models. Generally, conducted studies can be divided into spontaneousgenetic, inducible, transgenic and knockout models [27].

In the first group, two spontaneous mutations appeared in mice (Tight skin 1 mouse model and Tight skin 2 mouse model) and one in the chicken (UCD-200 chicken model) [28-30]. All of them were particularly useful in studying fibrosis and vasculopathy; however, they were limited by the fact that SSc is not hereditary. It is worth noting that out of all three known spontaneous genetic animal models, the UCD-200 model represented SSc most closely in terms of its pathological features. Therefore it is the most promising for future application.

In 2012 Inaoki et al. [31] studied a bleomycin mouse model focused on inducing fibrosis. It represented great scientific value, because it allowed study of the initial stages of fibrosis. However, the induction process was limited by the small number of chemicals to work with and difficulties with sclerodermatous graft versus host disease. Although it could replicate some of the earliest signaling patterns of SSc, it did not present the profound autoantibody patterns typical for this disorder. Other models worth mentioning are the hypochlorous model of SSc and the angiotensin II-inducible model of fibrosis [32, 33].

Transgenic models of fibrosis played an essential role in understanding how the specific genes contribute to the process of fibrosis [34-36]. Moreover, these models were also used in other forms of fibrosis [37]. Finally, the knockout models were used to visualize the effects of the presence or deletion of specific genes involved in the fibrosis process.

In the case of the SSc, other models of similar pathologies, such as idiopathic pulmonary fibrosis, were used [38]. These models were useful in studying the initiating events, genes and various factors required for fibrosis, because usually these features could not be considered in the patients.

Animal models provided insights into the vasculopathy, the role of proteins regulating fibrosis, and the innate immune system's role in initiating fibrotic events. All these models have also proved useful in increasing our understanding of SSc's pathogenesis and led to the development of various drugs; however, many of them did not translate well into therapeutic usage. Although 
all of them provided significant insight into a better understanding of rheumatological diseases, none of them could perfectly replicate all aspects and factors of an autoimmune disease.

\section{Clinical data on mesenchymal stem cells- based treatment in systemic sclerosis}

The variety of MSCs leads to the development of different therapies, and diverse case reports have been reported so far. Stem cell transplantation studies were shown to be effective in international multicenter clinical trials and demonstrated a sustained improvement in clinical effects [39, 40]. In 2008 the BM-MSCs usage in the treatment of progressive diffuse SSC was reported [41].

There, a 41-year-old female patient, after experiencing Raynaud's syndrome, was diagnosed with SSc. The patient underwent intravenous administration of MSCs per kilogram of body weight. During and after the treatment, the patient was observed, and within the first year no subjective complaints or objective organ dysfunctions were seen. The only exception was a common cold, which was quickly resolved without need for hospitalization. The patient experienced an improvement in her condition, as less stiffness in tendons and muscles was reported. Six months after transplantation, vascular ultrasound revealed an increase in the blood circulation of hands and fingers. There was a notable reduction in peripheral vascular resistance, as well [41].

Overall, this case represents encouraging and supportive data for MSCs to be used against severe SSc and other autoimmune diseases in general. Other similar therapies, supporting the effectiveness of the previous treatment, were later reported in 2011 [42]. The gathered data illustrated how MSCs transplantations can reduce vasculopathy and skin fibrosis and become a possible treatment for patients with refractory autoimmune disease. However, they also pointed out the necessity for phase II trials of MSCs transplantations.

Another study from 2013 was designed to assess the availability and efficacy of cell-based therapy with adipose-derived stem cells [43]. The research focuses on adipose tissue, as in the authors' opinion, ASCs represent a practical and appealing source of autologous stem cells, similar to BM-MSCs in many aspects [44].

However, the clinical use of ASCs seemed to be more advantageous since they can be obtained by a less invasive and uncomfortable method. The results of the treatment showed a significant improvement in six patients' health. All of them were followed postoperatively for one year. Medical assessments were performed weekly during the first month of observation and later monthly. The results presented a positive outcome where all of the patients showed the arrest of local disease progression and an overall improvement in wellness of the skin.

In light of that study, the clinical use of ASCs seems to be more advantageous to that of BM-MSCs. Not only can these cells be obtained in a less invasive and painful procedure, but also the frequency of stem cells in adipose tissue is higher than in the bone marrow. In addition, ASCs can longer retain their stem cell phenotypes and mesenchymal multipotency, which was observed to increase and was higher than in BM-MSCs' number of passages [45].

These findings can become a great asset in creating new therapeutic strategies in the treatment of SSc and other skin diseases. Fat tissue grafting has become a new approach in treating skin atrophy or fibrosis.

One of the first reports on this subject was published in 2014, where the implantation of fat was performed in patients presenting Raynaud's phenomenon [46]. A total of 13 patients were treated, and findings showed improvements in their health, such as reduced pain and improved skin and soft-tissue texture. However, it is worth noting that three patients revealed no changes. There were no significant complications, which suggests that fat grafting may benefit patients with skin diseases.

One year later, another study reported a substantial improvement in patients with SSc, who underwent injection of adipose tissue-derived fractions into their fingers [47]. Fifteen patients were included in this study, where eight of them had the limited, and the rest had the diffuse variant of the disease. Notable ulcer healing was observed in all of the patients who underwent the treatment, as well as pain relief in treated fingers. Therefore, the study suggests that autologous adipose tissuederived cell (ATDC) fractions might be a successful healing solution in SSc. That positive outcome certainly requires confirmation in more extensive studies.

In 2015 Granel et al. [48] demonstrated how injections of autologous stromal vascular fraction (SVF) from adipose tissue into the fingers of patients with SSc improve their quality of life. In all twelve patients who enrolled in this therapy, a significant improvement in pain reduction and grasping capacity was observed after six months, and improvement in skin sclerosis in the one-year follow-up.

Although that study was performed on a limited number of patients, it presented an innovative treatment. Later in 2017, a long-term follow-up beyond the end of the trial of that therapy was reported [49]. The findings illustrated a sustained improvement in patients' health. Tolerability, safety and efficacy were very promising. Future studies might help in a better understanding of the mechanisms supporting the clinical effects of stromal vascular fraction. 
Table I. Summary of clinical trials using mesenchymal stem cells for treatment of systemic sclerosis

\begin{tabular}{|c|c|c|c|}
\hline Type of treatment & No. of patients & Clinical outcome & References \\
\hline $\begin{array}{l}\text { Allogeneic BM-MSCS } \\
\text { (intravenous administration) }\end{array}$ & 1 & $\begin{array}{l}\text { Reduction of pain and ulcerations, } \\
\text { improvement of hand vasculopathy }\end{array}$ & 41 \\
\hline $\begin{array}{l}\text { Allogeneic BM-MSCS } \\
\text { (intravenous administration) }\end{array}$ & 5 & Improvement of skin fibrosis and vasculopathy & 42 \\
\hline $\begin{array}{l}\text { Autologous ASCs } \\
\text { (subcutaneous administration) }\end{array}$ & 6 & $\begin{array}{l}\text { Stabilization for all patients } \\
\text { and improvementof skin elasticity }\end{array}$ & 43 \\
\hline $\begin{array}{l}\text { Autologous fat grafting } \\
\text { (subcutaneous administration) }\end{array}$ & 13 & Improvement of perfusion and decreased number of ulcers & 46 \\
\hline $\begin{array}{l}\text { Autologous fat grafting } \\
\text { (subcutaneous administration) }\end{array}$ & 15 & Improvement of hand grasping, digital ulcerations and pain & 47 \\
\hline $\begin{array}{l}\text { Autologous SVF } \\
\text { (subcutaneous administration) }\end{array}$ & 12 & $\begin{array}{c}\text { Improvement of pain, grasping capacity, finger edema, } \\
\text { Raynaud's phenomenon }\end{array}$ & 48,49 \\
\hline $\begin{array}{l}\text { Allogeneic BM-MSCS } \\
\text { (intramuscular injection) }\end{array}$ & 20 & Results not provided yet & 50 \\
\hline
\end{tabular}

ASCs - adipose-derived stem cells, BM-MSCs - bone marrow-derived mesenchymal stem cells, SVF-stromal vascular fraction.

Finally, in November 2018 a clinical trial focused on using MSCs as a treatment for digital ulcers in SSC (MANUS) started [50]. The Mesenchymal stromal cells for Angiogenesis and Neovascularization in digital UIcers of Systemic sclerosis (MANUS) Trial is a randomized double-blind, placebo-controlled clinical trial, which aims to investigate the feasibility, safety and potential clinical effects of intramuscular injection of allogeneic BM-MSCs in a group of 20 participants. The study completion date is estimated to be in November 2020. The results may provide the basis for studies in other groups of patients with vascular and/or autoimmune diseases.

Stem cells are undoubtedly a great hope for the treatment of SSc due to their unique features, such as their availability, immunomodulatory properties, and the lack of ethical issues resulting from their use. Most of the studies conducted show that single transplantation of MSCs is safe and does not induce an immune response. However, repeated administration of MSCs may result in the production of allo-antibodies [51]. More research is needed regarding the potential risks, and long-term studies will be necessary to investigate the negative effects of these therapies.

Based on gathered data, the clinical use of MSCS seems to be a promising tool in regenerative medicine in the future (Table I).

\section{Conclusions}

Mesenchymal stem cell-based therapy represents a potential hope for patients with SSc, as many studies have now established the beneficial effects of administration of MSCs from different tissue sources. Mesenchymal stem cells require extreme care and a high level of competence.
Each step, from cell isolation to clinical trial evaluation, requires a deep understanding of their biology. Both autologous and allogeneic MSCs from BM or adipose tissue are being assessed and are subject to debate as to whether the benefits outweigh the potential losses.

Despite that, the properties of MSCs pique a lot of interest and enthusiasm from clinicians and researchers. Concerning the use of MSCs for clinical applications, further investigation of their functional properties is needed.

Future studies should focus on novel functional tests and potency assays to create clinically useful, validated, standardized, and safe therapy. The selection of the highest quality cells should also be taken into consideration to enhance the therapeutic potential of MSCs. Many questions remain unanswered; however, it is becoming clear that MSCs-based therapy may become a safe and efficient therapeutic option for patients with SSc.

The authors declare no conflict of interest.

\section{References}

1. Gabrielli A, Avvedimento EV, Krieg T. Scleroderma. N Engl J Med 2009; 360: 1989-2003, DOI: 10.1056/NEJMra0806188.

2. Badea I, Taylor M, Rosenberg A, Foldvari M. Pathogenesis and therapeutic approaches for improved topical treatment in localized scleroderma and systemic sclerosis. Rheumatology (Oxford) 2009; 48: 213-221, DOI: 10.1093/rheumatology/ken405.

3. Bergamasco A, Hartmann N, Wallace L, Verpillat P. Epidemiology of systemic sclerosis and systemic sclerosis-associated interstitial lung disease. Clin Epidemiol 2019; 11: 257-273, DOI: 10.2147/CLEP.S191418.

4. van den Hoogen F, Khanna D, Fransen J, et al. 2013 classification criteria for systemic sclerosis: an American College of Rheumatology/European League Against Rheumatism colla- 
borative initiative. Arthritis Rheum 2013; 65: 2737-2747, DOI: 10.1002/art.38098.

5. Silver RM. Clinical aspects of systemic sclerosis (scleroderma). Ann Rheum Dis 1991; 50 (Suppl 4): 854-861, DOI: 10.1136/ ard.50.Suppl_4.854.

6. Rozier P, Maria A, Goulabchand R, et al. Mesenchymal stem cells in systemic sclerosis: allogenic or autologous approaches for therapeutic use? Front Immunol 2018; 9: 2938, DOI: 10.3389/ fimmu.2018.02938.

7. Elhai M, Meune C, Boubaya M, et al. Mapping and predicting mortality from systemic sclerosis. Ann Rheum Dis 2017; 76 1897-1905, DOI: 10.1136/annrheumdis-2017-211448.

8. Zeineddine N, El Khoury L, Mosak J. Systemic sclerosis and malignancy: a review of current data. J Clin Med Res 2016; 8 625-632, DOI: 10.14740/jocmr2606w.

9. Katsiari CG, Simopoulou T, Alexiou I, Sakkas LI. Immunotherapy of systemic sclerosis. Hum Vaccines Immunother 2018; 14: 2559-2567, DOI: 10.1080/21645515.2018.1491508.

10. Ullah I, Subbarao RB, Rho GJ. Human mesenchymal stem cells - current trends and future prospective. Biosci Rep 2015; 35: e00191, DOI: 10.1042/BSR20150025.

11. Cras A, Farge D, Carmoi T, et al. Update on mesenchymal stem cell-based therapy in lupus and scleroderma. Arthritis Res Ther 2015; 17: 301, DOI: 10.1186/s13075-015-0819-7.

12. Paduano F, Marrelli M, Amantea M, et al. Adipose tissue as a strategic source of mesenchymal stem cells in bone regeneration: a topical review on the most promising craniomaxillofacial applications. Int J Mol Sci 2017; 18: 2140, DOI: 10.3390/ ijms18102140.

13. Giai Via A, Frizziero A, Oliva F. Biological properties of mesenchymal stem cells from different sources. Muscles Ligaments Tendons J 2012; 2: 154-162.

14. Lee JS, Hong JM, Moon GJ, et al. A long-term follow-up study of intravenous autologous mesenchymal stem cell transplantation in patients with ischemic stroke. Stem Cells 2010; 28 : 1099-1106, DOI: 10.1002/stem.430.

15. Lalu MM, McIntyre L, Pugliese C, et al. Safety of cell therapy with mesenchymal stromal cells (SafeCell): a systematic review and meta-analysis of clinical trials. PLoS One 2012; 7: e47559, DOI: 10.1371/journal.pone.0047559.

16. Stefanis AJ, Groh T, Arenbergerova M, et al. Stromal vascular fraction and its role in the management of alopecia: a review. Clin Aesthet Dermatol 2019; 12: 35-44.

17. Bourin P, Bunnell BA, Casteilla L, et al. Stromal cells from the adipose tissue-derived stromal vascular fraction and culture expanded adipose tissue-derived stromal/stem cells: a joint statement of the International Federation for Adipose Therapeutics and Science (IFATS) and the International Society for Cellular Therapy (ISCT). Cytotherapy 2013; 15: 641-648, DOI: 10.1016/j.jcyt.2013.02.006.

18. Rehman J, Traktuev D, Li J, et al. Secretion of angiogenic and antiapoptotic factors by human adipose stromal cells. Circulation 2004; 109: 1292-1298, DOI: 10.1161/01.CIR.0000121425.42966.F1.

19. Peltzer J, Aletti M, Frescaline N, et al. Mesenchymal stromal cells based therapy in systemic sclerosis: Rational and challenges. Front Immunol 2018; 9: 2013, DOI: 10.3389/fimmu. 2018.02013.
20. Lo Sicco C, Reverberi D, Balbi C, et al. Mesenchymal stem cell-derived extracellular vesicles as mediators of anti-inflammatory effects: endorsement of macrophage polarization. Stem Cells Transl Med 2017; 6: 1018-1028, DOI: 10.1002/sctm. 16-0363.

21. Musiat-Wysocka A, Kot M, Majka M. The pros and cons of mesenchymal stem cell-based therapies. Cell Transplant 2019; 28: 801-812, DOI: 10.1177/0963689719837897.

22. Hatzistergos KE, Blum A, Ince T, et al. What is the oncologic risk of stem cell treatment for heart disease? Circ Res 2011; 108: 1300-1303, DOI: 10.1161/CIRCRESAHA.111.246611.

23. Barkholt L, Flory $E$, Jekerle $\mathrm{V}$, et al. Risk of tumorigenicity in mesenchymal stromal cell-based therapies - bridging scientific observations and regulatory viewpoints. Cytotherapy 2013; 15: 753-759, DOI: 10.1016/j.jcyt.2013.03.005.

24. Herberts CA, Kwa MS, Hermsen HP. Risk factors in the development of stem cell therapy. J Transl Med 2011; 9: 29, DOI: 10.1186/1479-5876-9-29.

25. Clarke MR, Imhoff FM, Baird SK. Mesenchymal stem cells inhibit breast cancer cell migration and invasion through secretion of tissue inhibitor of metalloproteinase- 1 and -2 . Mol Carcinog 2015; 54: 1214-1219, DOI: 1 0.1002/mc.22178.

26. Bruno S, Collino F, lavello A, Camussi G. Effects of mesenchymal stromal cell-derived extracellular vesicles on tumor growth. Front Immunol 2014; 5: 382, DOI: 10.3389/fimmu.2014.00382.

27. Artlett CM. Animal models of systemic sclerosis: their utility and limitations. Open Access Rheumatol 2014; 6: 65-81, DOI: 10.2147/OARRR.S50009.

28. Wipff J, Giraud M, Sibilia J, et al. Polymorphic markers of the fibrillin-1 gene and systemic sclerosis in European Caucasian patients. J Rheumatol 2008; 35: 643-649.

29. Gentiletti J, McCloskey LJ, Artlett CM, et al. Demonstration of autoimmunity in the tight skin-2 mouse: a model for scleroderma. J Immunol 2005; 175: 2418-2426, DOI: 10.4049/jimmunol. 175.4.2418.

30. Nguyen VA, Sgonc R, Dietrich H, Wick G. Endothelial in jury in internal organs of University of California at Davis line 200 (UCD 200) chickens, an animal model for systemic sclerosis (Scleroderma). J Autoimmun 2000; 14: 143-149, DOI: 10.1006/ jaut.1999.0355.

31. Inaoki M, Kawabata C, Nishijima C, et al. Case of bleomycininduced scleroderma. J Dermatol 2012; 39: 482-484, DOI: 10.1111/j.1346-8138.2011.01301.x.

32. Batteux F, Kavian N, Servettaz A. New insights on chemically induced animal models of systemic sclerosis. Curr Opin Rheumatol 2011; 23: 511-518, DOI: 10.1097/BOR.0b013e32834b1606.

33. Stawski L, Han R, Bujor AM, Trojanowska M. Angiotensin II induces skin fibrosis: a novel mouse model of dermal fibrosis. Arthritis Res Ther 2012; 14: R194, DOI: 10.1186/ar4028.

34. Richard V, Solans V, Favre J, et al. Role of endogenous endothelin in endothelial dysfunction in murine model of systemic sclerosis: tight skin mice 1. Fundam Clin Pharmacol 2008; 22 : 649-655, DOI: 10.1111/j.1472-8206.2008.00634.x.

35. Maurer B, Busch N, Jüngel A, et al. Transcription factor fosrelated antigen-2 induces progressive peripheral vasculopathy in mice closely resembling human systemic sclerosis. Circulation 2009; 120: 2367-2376, DOI: 10.1161/CIRCULATIONAHA.109.855114. 
36. Maurer B, Distler JH, Distler O. The Fra-2 transgenic mouse model of systemic sclerosis. Vascul Pharmacol 2013; 58: 194-201, DOI: 10.1016/j.vph.2012.12.001

37. Hocher B, Schwarz A, Fagan KA, et al. Pulmonary fibrosis and chronic lung inflammation in ET-1 transgenic mice. Am J Respir Cell Mol Biol 2000; 23: 19-26, DOI: 10.1165/ajrcmb.23.1.4030.

38. Gasse P, Mary C, Guenon I, et al. IL-1R1/MyD88 signaling and the inflammasome are essential in pulmonary inflammation and fibrosis in mice. J Clin Invest 2007; 117: 3786-3799, DOI: $10.1172 / \mathrm{JCl} 32285$.

39. Burt RK, Shah SJ, Dill K, et al. Autologous non-myeloablative haemopoietic stem-cell transplantation compared with pulse cyclophosphamide once per month for systemic sclerosis (ASSIST): an open-label, randomised phase 2 trial. Lancet 2011; 378: 498-506, DOI: 10.1016/S0140-6736(11)60982-3.

40. Burt RK, Farge D. Systemic sclerosis: autologous HSCT is efficacious, but can we make it safer? Nat Rev Rheumatol 2018, 14: 189-191, DOI: 10.1038/nrrheum.2018.34.

41. Christopeit M, Schendel M, Föll J, et al. Marked improvement of severe progressive systemic sclerosis after transplantation of mesenchymal stem cells from an allogeneic haploidentical-related donor mediated by ligation of CD137L. Leukemia 2008; 22: 1062-1064, DOI: 10.1038/sj.leu.2404996.

42. Keyszer G, Christopeit M, Fick S, et al. Treatment of severe progressive systemic sclerosis with transplantation of mesenchymal stromal cells from allogeneic related donors: report of five cases. Arthritis Rheum 2011; 63: 2540-2542, DOI: 10.1002/art.30431.

43. Scuderi N, Ceccarelli S, Onesti MG, et al. Human adiposederived stromal cells for cell-based therapies in the treatment of systemic sclerosis. Cell Transplant 2013; 22: 779-795, DOI: 10.3727/096368912X639017.

44. Zuk PA, Zhu M, Ashjian P, et al. Human adipose tissue is a source of multipotent stem cells. Mol Biol Cell 2002; 13 4279-4295, DOI: 10.1091/mbc.E02-02-0105.
45. Zhu Y, Liu T, Song K, et al. Adipose-derived stem cell: a better stem cell than BMSC. Cell Biochem Funct 2008; 26: 664-675, DOI: 10.1002/cbf.1488.

46. Bank J, Fuller SM, Henry GI, Zachary LS. Fat grafting to the hand in patients with Raynaud phenomenon: a novel therapeutic modality. Plast Reconstr Surg 2014; 133: 1109-1118, DOI: 10.1097/PRS.0000000000000104.

47. Papa N Del, Di Luca G, Sambataro D, et al. Regional implantation of autologous adipose tissue-derived cells induces a prompt healing of long-lasting indolent digital ulcers in patients with systemic sclerosis. Cell Transplant 2015; 24: 22972305, DOI: 10.3727/096368914X685636.

48. Granel B, Daumas A, Jouve E, et al. Safety, tolerability and potential efficacy of injection of autologous adipose-derived stromal vascular fraction in the fingers of patients with systemic sclerosis: an open-label phase I trial. Ann Rheum Dis 2015; 74: 2175-2182, DOI: 10.1136/annrheumdis-2014-205681.

49. Daumas A, Magalon J, Jouve E, et al. Long-term follow-up after autologous adipose-derived stromal vascular fraction injection into fingers in systemic sclerosis patients. Curr Res Transl Med 2017; 65: 40-43, DOI: 10.1016/j.retram.2016.10.006.

50. van Rhijn-Brouwer FC, Gremmels H, Fledderus JO, et al. A randomised placebo-controlled double-blind trial to assess the safety of intramuscular administration of allogeneic mesenchymal stromal cells for digital ulcers in systemic sclerosis: the MANUS Trial protocol. BMJ Open 2018; 8: e020479, DOI: 10.1136/bmjopen-2017-020479.

51. Cho PS, Messina DJ, Hirsh EL, et al. Immunogenicity of umbilical cord tissue-derived cells. Blood 2008; 111: 430-438, DOI: 10.1182/blood-2007-03-078774 